\title{
High Plant Density of Cucumber (Cucumis sativus L.) Seedlings Mitigates Inhibition of Photosynthesis Resulting from High Vapor-pressure Deficit
}

\author{
Toshio Shibuya ${ }^{1}$, Akihito Sugimoto, Yoshiaki Kitaya, and \\ Makoto Kiyota \\ Division of Graduate School of Life and Environmental Science, Osaka \\ Prefecture University, Gakuen-cho, Naka-ku, Sakai, Osaka 599-8531, Japan
}

Additional index words. boundary layer, leaf area index, leaf conductance, net photosynthetic rate, plant community, transpiration rate

\begin{abstract}
To evaluate the effects of plant density on gas exchanges under water stress resulting from high vapor-pressure deficit (VPD), we measured net photosynthetic rate $\left(P_{n}\right)$, transpiration rate, and leaf conductance $\left(g_{1}\right)$ of cucumber (Cucumis sativus $L_{\text {. }}$ ) seedlings before and after raising the VPD at different plant densities. Measurements were conducted continuously using a chamber and weighing method. Five, nine, or 12 seedlings with leaf area index (LAI) of $0.39,0.73$, and 1.10 , respectively, were placed in the assimilation chamber. The average VPD in the chamber was raised from 1.1 to 3.7 kPa 30 min after the starting measurement. The $P_{\mathrm{n}}$ and $g_{1}$ decreased after raising the VPD above the plant community from 1.1 to $3.7 \mathrm{kPa}$. The VPD near the leaf surface (measured with 3-mm diameter humidity sensors) decreased with increasing LAI of the plant community, whereas average VPD in the whole chamber did not change with LAI. We noted significant negative correlations between the VPD near the leaf surface and $\boldsymbol{P}_{\mathrm{n}}$ and $g_{1}$. These results indicate that higher plant density mitigates the inhibition of photosynthesis resulting from high VPD by maintaining a lower VPD near the leaf surface with the development of a thicker boundary layer above the canopy.
\end{abstract}

In a community with high plant density, as seen in transplant production, the gas exchanges between plant and atmosphere are conducted through a unique boundary layer developed above the canopy (Kim et al., 1996b). The boundary layer varies with changes in the structure of the plant community and microclimatic parameters such as air velocity. The variation of the boundary layer, in turn, affects microclimatic parameters in the plant community such as air temperature, relative humidity, air velocity, and $\mathrm{CO}_{2}$ concentration (Kim et al., 1996a, 1996b; Kitaya et al., 1998), thereby affecting the gas exchange of the plant community and its consequent growth (Kitaya et al., 2000, 2002, 2004; Shibuya and Kozai, 1998, 2001). Generally, $\mathrm{CO}_{2}$ supply into the canopy is inhibited with increasing plant density (Shibuya and Kozai, 2001; Yabuki et al., 1967) resulting from the thicker boundary layer that develops above the canopy and the decline of the $\mathrm{CO}_{2}$ concentration and air velocity in the canopy (Kim et al., 1996a, 1996b; Kitaya et al., 1998). On the other hand, increasing plant density may enhance $\mathrm{CO}_{2}$ exchange of the leaf when the

Received for publication 21 Jan. 2009. Accepted for publication $24 \mathrm{Feb} .2009$.

This study was partially supported by the Ministry of Education, Science, Sports and Culture of Japan Grant-in-aid for young scientists (A), 15688007, 2003-2005.

${ }^{1}$ To whom reprint requests should be addressed; e-mail shibuya@envi.osakafu-u.ac.jp.
$\mathrm{CO}_{2}$ supply to plants is limited by a decrease in leaf conductance resulting from water stress resulting from high vapor-pressure deficit (VPD). Under higher plant density, the VPD near the leaf surface decreases as a result of the thicker boundary layer (Kim et al., 1996b; Kitaya et al., 1998), which inhibits water vapor exchange between plants and the atmosphere. The gas exchanges at the leaf surface are inhibited by a decrease in leaf conductance with stomatal closing when the VPD near the leaf surface increases (Bunce, 1984; Shibuya et al., 2003, 2006; Woledge et al., 1989). In such a case, high plant density would decrease the VPD near the leaf surface with development of the boundary layer above the plant canopy, thereby likely mitigating the inhibition of $\mathrm{CO}_{2}$ exchange resulting from the water stress.

In this study, we measured changes in net photosynthetic rate, transpiration rate, and leaf conductance of a seedling community after raising the VPD at various plant densities to evaluate the effects of plant density on gas exchanges under the water stress resulting from high VPD. We developed a new technique to measure leaf conductance continuously based on weight of the plant community and absolute humidity near the leaf surface, which was measured with tiny humidity sensors.

\section{Materials and Methods}

Plant material. Cucumber (Cucumis sativus L. 'Hokushin') seedlings grown in a growth chamber for $12 \mathrm{~d}$ after seeding were used. The growth conditions were $30 / 26{ }^{\circ} \mathrm{C}$ (light/dark) air temperatures, $50 \%$ to $60 \%$ relative humidity, and a photosynthetic photon flux $(P P F)$ density of $500 \mu \mathrm{mol} \cdot \mathrm{m}^{-2} \cdot \mathrm{s}^{-1}$ during a photoperiod of $12 \mathrm{~h}$. The seedlings were grown in plastic pots $(60-\mathrm{mm}$ diameter, $55-\mathrm{mm}$ high) with vermiculite medium. Nutrient solution (A-type recipe of Otsuka House Solution, diluted by $1 / 2$; Otsuka Chemical Co., Ltd., Osaka, Japan) was supplied from the bottom of the pot as necessary. The seedlings had one foliage leaf (length: 60 to $70 \mathrm{~mm}$ ) and a pair of cotyledons.

Measuring conditions. Measurements of net photosynthetic rate, transpiration rate, and leaf conductance of the seedlings were conducted in an assimilation chamber [length $(\mathrm{L}) \times$ depth $(\mathrm{D}) \times$ height $(\mathrm{H}): 650 \times 400 \times 400$ $\mathrm{mm}$ ] containing a wind tunnel (Fig. 1). Five, nine, or 12 seedlings were placed in the wind tunnel $(\mathrm{L} \times \mathrm{D} \times \mathrm{H}: 500 \times 300 \times 300 \mathrm{~mm})$ without intershading between seedlings. The measurements were conducted with two replications for the three plant densities. The different seedlings were used for each measurement. The surface of the growing medium was covered with a plastic film to prevent evaporation. The air velocity windward was controlled at $0.4 \mathrm{~m} \cdot \mathrm{s}^{-1}$ with fans in the wind tunnel. Air temperature and VPD of the assimilation chamber were controlled by introducing controlled air from air conditioning systems connected with the chamber. A blower was used for introducing the controlled air. We used two air conditioning systems to maintain low or high VPD. The assimilation chamber was connected with the low-VPD system at the beginning of the measurement. The VPD in the assimilation chamber was raised by changing the connection to the highVPD system 30 min after the starting measurement, and then a measurement was conducted for $90 \mathrm{~min}$. The air flow rates of the assimilation chamber were 0.33 and 0.22 $\mathrm{m}^{3} \cdot \mathrm{min}^{-1}$ under low and high VPD, respectively. Continuous illumination was provided during the measurements with white fluorescent lamps (FPL55EX-N; Matsushita Electric Industrial Co., Ltd., Kadoma, Japan) at a $P P F$ density of $500 \mu \mathrm{mol} \cdot \mathrm{m}^{-2} \cdot \mathrm{s}^{-1}$. The $\mathrm{CO}_{2}$ concentration in the chamber was maintained at $\approx 400 \mu \mathrm{mol} \cdot \mathrm{mol}^{-1}$ without controls.

Measurement method. The weight of the plant community was measured with an electric balance (BW4200; Shimadzu Co., Ltd., Kyoto, Japan) placed below the plant community. The absolute humidity and air temperature near the leaf surface was measured with tiny humidity sensors (RHM1000S; Ricoh Elemex Co., Ltd., Nagoya, Japan). The probes (3-mm diameter, 20-mm long) of the sensor were placed $3 \mathrm{~mm}$ above and below the center of the foliage leaf of the central seedling of the community (Fig. 1). Leaf temperature was measured by contacting a junction of a thermocouple (T-type, 0.1$\mathrm{mm}$ diameter) on the abaxial leaf surface. The vapor pressure near the leaf surface was estimated from the absolute humidity. The VPD near the leaf surface was estimated by 
(A)

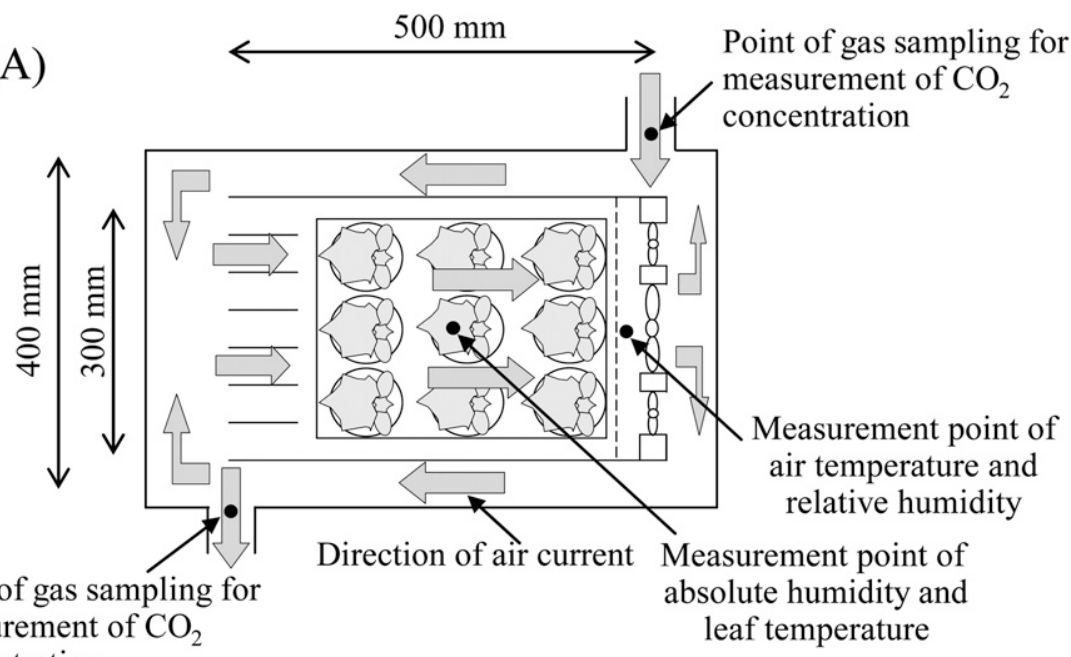

Point of gas sampling for measurement of $\mathrm{CO}_{2}$ concentration

(B)

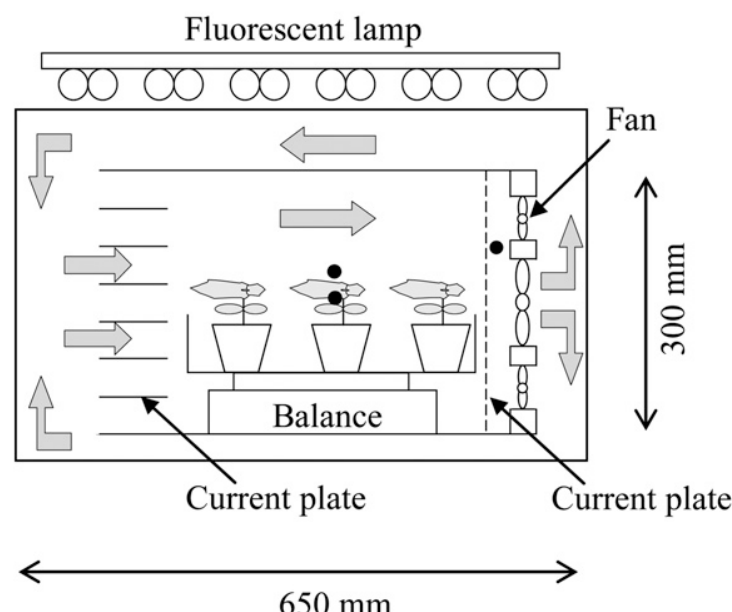

Fig. 1. Schematic diagram of the experimental assimilation chamber with wind tunnel: (A) horizontal section; (B) cross-section.

subtracting the vapor pressure near the leaf surface from saturating vapor pressure at the leaf temperature. The $\mathrm{CO}_{2}$ concentration at the inlet and outlet of the assimilation chamber was sampled alternately at 1-min intervals with an air pump at a flow rate of 1.0 $\mathrm{L} \cdot \mathrm{min}^{-1}$; samples were measured with an infrared gas analyzer (LI-7000; LI-COR Inc., Lincoln, NE). Effects of air leak of the chamber were neglected, because air pressure in the chamber was maintained positive by introducing air with the blower.

The average air temperature and relative humidity in the assimilation chamber were measured with a temperature and humidity recorder (TR-72s; T\&D Co. Ltd., Matsumoto, Japan) placed leeward of the wind tunnel. The average VPD in the assimilation chamber was estimated based on air temperature and relative humidity. The air velocity at $5 \mathrm{~mm}$ above the leaf surface under each plant density condition was measured with a hot-wire anemometer (Anemomaster Model 6071; Kanomax Japan, Inc., Suita, Japan) at the end of each set of measurements.

Leaf area index (LAI) of the plant community was estimated based on the canopy floor area and the total leaf area measured at the end of each experiment. The average LAI values at plant densities of five, nine, and 12 plants per chamber (low, middle, and high plant density) were $0.39,0.73$, and 1.10 , respectively.

Calculations. The transpiration rate per leaf area of the plant community $(T r$, $\mathrm{mol} \cdot \mathrm{m}^{-2} \cdot \mathrm{s}^{-1} \mathrm{H}_{2} \mathrm{O}$ ) was estimated based on the change in weight of the plant community with the equation:

$$
\operatorname{Tr}=\frac{W_{1}-W_{2}}{t_{2}-t_{1}} \cdot \frac{1}{m_{\mathrm{v}}} \cdot \frac{1}{A}
$$

where $W_{1}$ and $W_{2}(\mathrm{~g})$ were the weights of the plant community at times $t_{1}$ and $t_{2}(\mathrm{~s})$, respectively, $m_{\mathrm{v}}$ was the molecular weight of water $\left(18 \mathrm{~g} \cdot \mathrm{mol}^{-1} \mathrm{H}_{2} \mathrm{O}\right)$, and $A$ was total leaf area of the plant community $\left(\mathrm{m}^{2}\right)$.

The total vapor diffusion resistance $\left(R_{1+\mathrm{b}}\right.$, $\mathrm{s} \cdot \mathrm{m}^{-1}$ ) from inside the leaf to the atmosphere, accounting for the vapor diffusion resistance of leaf $\left(R_{1}, \mathrm{~s} \cdot \mathrm{m}^{-1}\right)$ and leaf boundary layer resistance from leaf surface to $\left(R_{\mathrm{b}}, \mathrm{s} \cdot \mathrm{m}^{-1}\right)$, was estimated based on the diffusion vapor model (Monteith and Unsworth, 2008) using the equation:

$$
R_{1+\mathrm{b}}=R_{1}+R_{\mathrm{b}}=\frac{y_{1}-y_{\mathrm{a}}}{m_{\mathrm{v}} \cdot 0.5 T r}
$$

where $y_{\mathrm{a}}$ and $y_{1}\left(\mathrm{~g} \cdot \mathrm{m}^{-3} \mathrm{H}_{2} \mathrm{O}\right)$ were the absolute humidity near the leaf surface and inside the leaf, respectively, and $0.5 \operatorname{Tr}\left(\mathrm{mol} \cdot \mathrm{m}^{-2} \cdot \mathrm{s}^{-1} \mathrm{H}_{2} \mathrm{O}\right)$ the transpiration rate per total leaf surface area. $y_{\mathrm{a}}$ was determined based on the average of the absolute humidity above and below the leaf surface. $y_{1}$ was assumed to be the saturated absolute humidity at the leaf temperature, because the intercellular space in the leaf was assumed to be saturated with water vapor. In this study, $y_{\mathrm{a}}$ and $y_{1}$ measured at the center of the foliage leaves were taken with two to three replications as the representative values.

The $R_{\mathrm{b}}$ value was determined using a model plant community. The leaf of the model plant was made with a regular pentagon-shaped piece of wet paper ( $60 \mathrm{~mm}$ long; Toyo Roshi Kaisha, Ltd., Tokyo, Japan). Transpiration rate $\left(\operatorname{Tr}_{\mathrm{m}}, \mathrm{mol} \cdot \mathrm{m}^{-2} \cdot \mathrm{s}^{-1} \mathrm{H}_{2} \mathrm{O}\right)$ of the model plant community, absolute humidity $\left(y_{\mathrm{a}}, \mathrm{g} \cdot \mathrm{m}^{-3} \mathrm{H}_{2} \mathrm{O}\right)$ near the model leaf surface, absolute humidity $\left(y_{\mathrm{ml}}, \mathrm{g} \cdot \mathrm{m}^{-3} \mathrm{H}_{2} \mathrm{O}\right)$ inside the model leaf, and leaf temperatures were measured using the same methods as in the cucumber plant community. The $R_{\mathrm{b}}$ was estimated with the equation:

$$
R_{\mathrm{b}}=\frac{y_{\mathrm{m}}-y_{\mathrm{a}}}{m_{\mathrm{v}} \cdot 0.5 T r_{\mathrm{m}}}
$$

The leaf conductance $\left(g_{1}, \mathrm{~m} \cdot \mathrm{s}^{-1}\right)$ was estimated with the equation:

$$
g_{1}=\frac{1}{R_{1}}=\frac{1}{R_{1+\mathrm{b}}-R_{\mathrm{b}}}
$$

The photosynthetic rate $\left(P_{\mathrm{n}}, \mathrm{mol} \cdot \mathrm{m}^{-2} \cdot \mathrm{s}^{-1}\right.$ $\mathrm{CO}_{2}$ ) of the plant community per leaf area was estimated with the equation:

$$
P_{\mathrm{n}}=v \cdot \frac{C_{\text {in }}-C_{\text {out }}}{A}
$$

where $C_{\text {in }}$ and $C_{\text {out }}\left(\mathrm{mol} \cdot \mathrm{m}^{-3} \mathrm{CO}_{2}\right)$ was the $\mathrm{CO}_{2}$ concentration at the inlet and outlet of the assimilation chamber, respectively, and $v$ $\left(\mathrm{m}^{3} \cdot \mathrm{s}^{-1}\right)$ was the air flow rate of the chamber.

Statistics. Relationships between the variables were analyzed by Pearson's productmoment correlation coefficient at $P=0.10$, 0.05 , and 0.01 .

\section{Results and Discussion}

The air velocity at $5 \mathrm{~mm}$ above the leaf surface decreased with increasing plant density with values of $0.27,0.17$, and $0.10 \mathrm{~m} \cdot \mathrm{s}^{-1}$, respectively, for low, middle, and high plant density. The $R_{\mathrm{b}}$ determined by using wet mode leaf was $7.3 \times 10^{-2}, 8.5 \times 10^{-2}$, and $9.5 \times 10^{-2} \mathrm{~s} \cdot \mathrm{mm}^{-1}$ for low, middle, and high plant density, respectively. The $R_{\mathrm{b}}$ tended to be decreased linearly with the increasing air velocity. The relationship between the air velocity and the $R_{\mathrm{b}}$ is similar to previous studies (Yabuki, 2004). This lower air velocity and $R_{\mathrm{b}}$ with higher plant density was caused by the development of a thicker boundary layer above the canopy. The time courses of average air temperature, relative humidity, and VPD in the assimilation chamber are shown in Figure 2. The air temperature was maintained at $30^{\circ} \mathrm{C}$. The relative humidity was maintained at $73 \%$ before raising the VPD and then decreased to $13 \%$ afterward. The VPD was maintained at $1.1 \mathrm{kPa}$ and then increased to $3.7 \mathrm{kPa}$. These values were nearly the same at all plant densities. The 

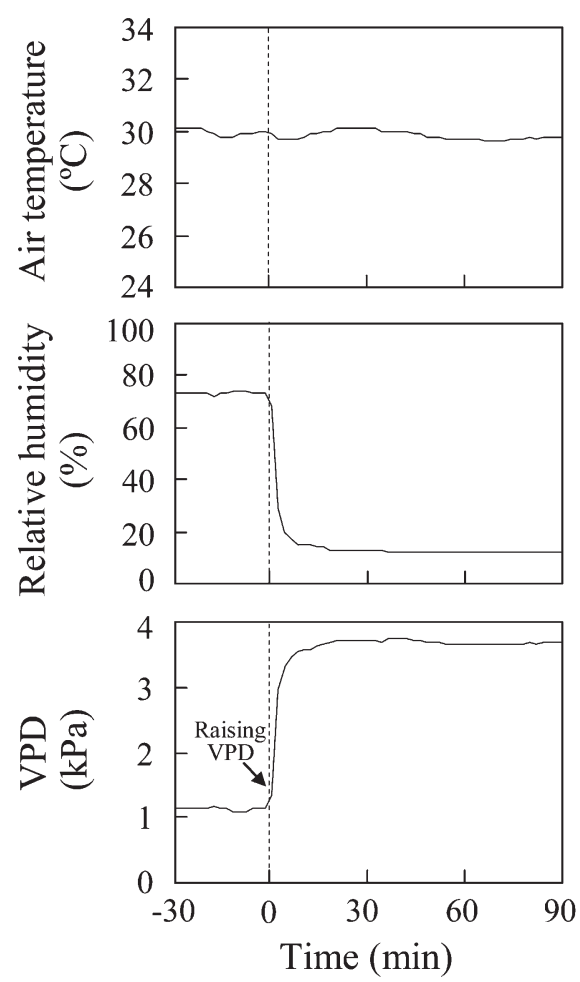

Fig. 2. Time courses of average air temperature, relative humidity, and vapor-pressure deficit (VPD) in the chamber before and after raising the VPD. Examples of the measurements at low plant density are shown.

average VPD values near the leaf surface before raising the VPD were $0.32,0.29$, and $0.29 \mathrm{kPa}$ at low, middle, and high plant density, respectively (Fig. 3). The higher VPD at lower plant density before raising the VPD was probably the result of a thinner boundary layer above the canopy. At 60 to 90 min after raising the VPD, the VPD near the leaf surface tended to decrease with increasing plant density with values of $1.96,1.52$, and $1.08 \mathrm{kPa}$, respectively, at low, middle, and high plant density (Fig. 3). The lower VPD near leaf surface of higher plant density was probably the result of a thicker boundary layer developing above the canopy, which would inhibit vapor diffusion. After raising the VPD, near the leaf surface, the VPD decreased with LAI $(P<0.05$; Fig. 4), whereas before the VPD was raised, there was no significant correlation with LAI.

The $T r$ increased for 10 min after raising the VPD and then decreased (Fig. 3). At 0 to 30 min after raising the VPD, the $\operatorname{Tr}$ decreased with increasing plant density with average values of $5.96,4.86$, and $4.52 \mathrm{mmol} \cdot \mathrm{m}^{-2} \cdot \mathrm{s}^{-1}$ $\mathrm{H}_{2} \mathrm{O}$, respectively, at low, middle, and high plant density. There was a significant negative correlation between LAI and $\operatorname{Tr} 0$ to 30 min after raising the VPD, indicating that water loss from the plant immediately after the VPD rise was greater at lower plant density. There was no significant correlation between LAI and $\operatorname{Tr} 60$ to $90 \mathrm{~min}$ after raising the VPD (Fig. 5).

The $g_{1}$ decreased after the VPD was raised (Fig. 3). At 60 to $90 \mathrm{~min}$ after raising the

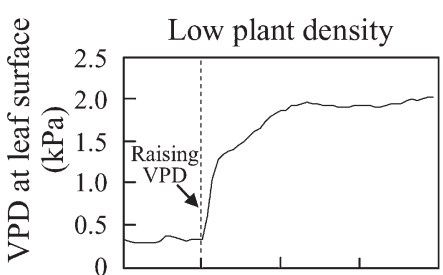

Middle plant density
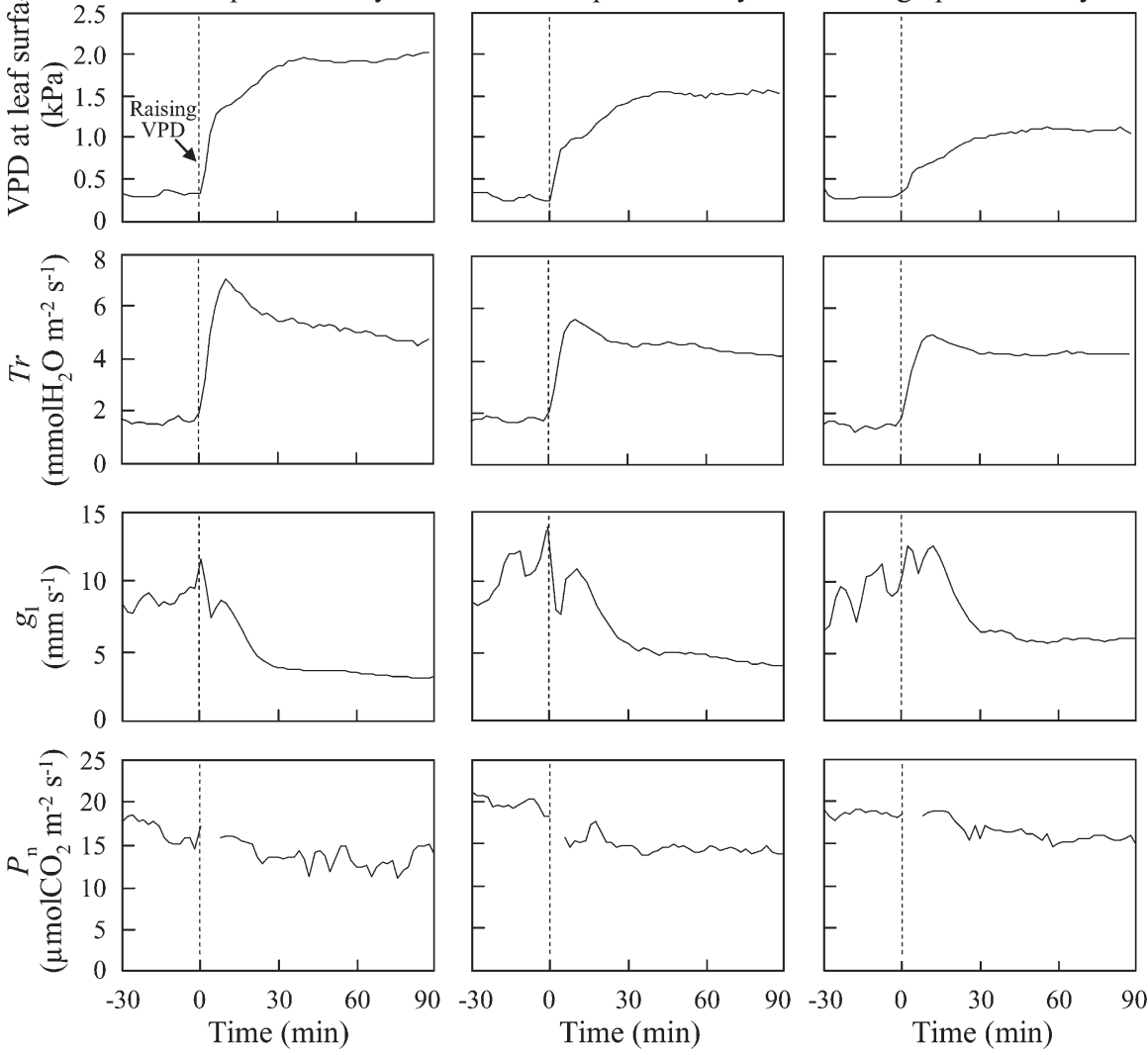

Fig. 3. Time courses of vapor-pressure deficit (VPD) near the leaf surface, transpiration rate (Tr), leaf conductance $\left(g_{1}\right)$, and net photosynthetic rate $\left(P_{\mathrm{n}}\right)$ of cucumber seedlings at different plant densities before and after raising the VPD. Averages of two replications are shown. Missing values in net photosynthetic rate are caused by disturbances of $\mathrm{CO}_{2}$ concentration.

VPD, the $g_{1}$ tended to increase with increasing plant density with average values of 3.5 , 4.2 , and $5.9 \mathrm{~mm} \cdot \mathrm{s}^{-1}$, respectively, at low, middle, and high plant density. The start of decrease in $g_{1}$ tended to be delayed for $10 \mathrm{~min}$ after raising the VPD under high plant density. The decrease in $g_{1}$ after the VPD rise was probably the result of stomatal closing caused by excessive transpiration at the high VPD, which was observed in a previous study (Shibuya et al., 2006). After raising the VPD, the $g_{1}$ increased with LAI $(P<0.10$; Fig. 5), whereas before the VPD was raised, there was no significant correlation with LAI.

The $P_{\mathrm{n}}$ decreased after raising the VPD (Fig. 3). At 60 to $90 \mathrm{~min}$ after the VPD rise, the $P_{\mathrm{n}}$ increased with plant density with average values of $13.0,14.2$, and 15.5 $\mu \mathrm{mol} \cdot \mathrm{m}^{-2} \cdot \mathrm{s}^{-1} \mathrm{CO}_{2}$, respectively, at low, middle, and high plant density. The decrease in $P_{\mathrm{n}}$ after raising the VPD was probably the result of the decrease in $g_{1}$. After raising the VPD, the $P_{\mathrm{n}}$ increased significantly with increasing LAI $(P<0.01$; Fig. 5), whereas before the VPD was raised, there was no significant correlation with LAI. The increase in thickness of boundary layer with LAI probably did not affect direct $\mathrm{CO}_{2}$ assimilation of the seedlings in this experiment, because $P_{\mathrm{n}}$ before raising VPD was not changed with LAI, which affected development of the boundary layer. This result indicates that $\mathrm{CO}_{2}$ concentration near the leaf

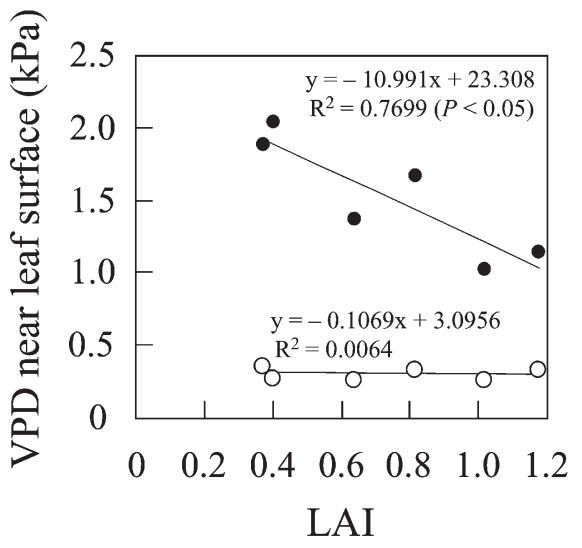

Fig. 4. Effects of leaf area index (LAI) on vaporpressure deficit (VPD) near the leaf surface of cucumber seedlings at low (average 0 to $30 \mathrm{~min}$ before raising VPD, open circles) and high VPD (average 60 to $90 \mathrm{~min}$ after raising VPD, closed circles).

surface was probably unaffected significantly by the thickness of the boundary layer as the VPD near the leaf surface.

There was no significant correlation between VPD near the leaf surface and $T r$ (Fig. 6). With increasing plant density, the $g_{1}$ varied inversely with VPD near the leaf surface; therefore, $\operatorname{Tr}$ (i.e., the product of $\mathrm{g}_{1}$ and VPD) probably did not change significantly. In other words, the direct effect of 

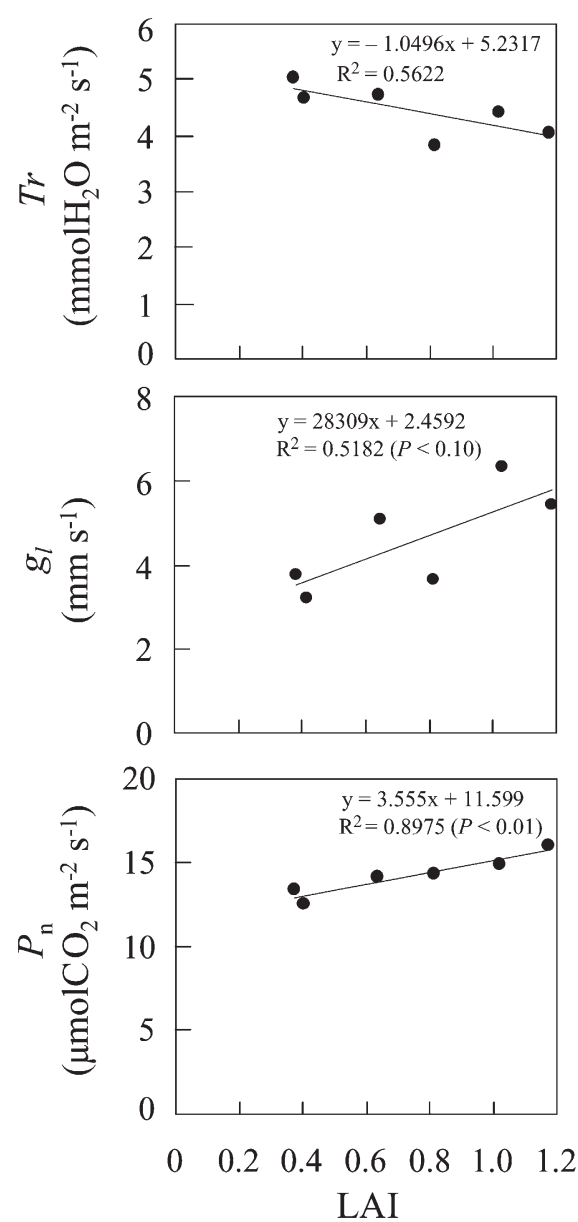

Fig. 5. Effects of leaf area index (LAI) on transpiration rate $(\operatorname{Tr})$, leaf conductance $\left(g_{1}\right)$, and net photosynthetic rate $\left(P_{\mathrm{n}}\right)$ of cucumber seedlings at high vapor-pressure deficit (average 60 to 90 min after raising VPD).

VPD on $T r$ and the indirect effect of VDP resulting from the change in $g_{1}$ compensated for each other in this study. In contrast, $P_{\mathrm{n}}$ and $g_{1}$ decreased with increasing VPD near the leaf surface (Fig. 6). There were significant negative correlations between VPD near the leaf surface and $P_{\mathrm{n}}$ and $g_{1}$ (both $P<$ $0.01)$. These results indicate that a higher plant density maintains a lower VPD near the leaf surface with development of the boundary layer above the canopy, which mitigates the decrease in $g_{1}$ after stomatal closure resulting from the excessive transpiration immediately after raising the VPD, thereby mitigating the inhibition of $P_{\mathrm{n}}$. The LAI of 0.39 to 1.10 examined in this experiment is
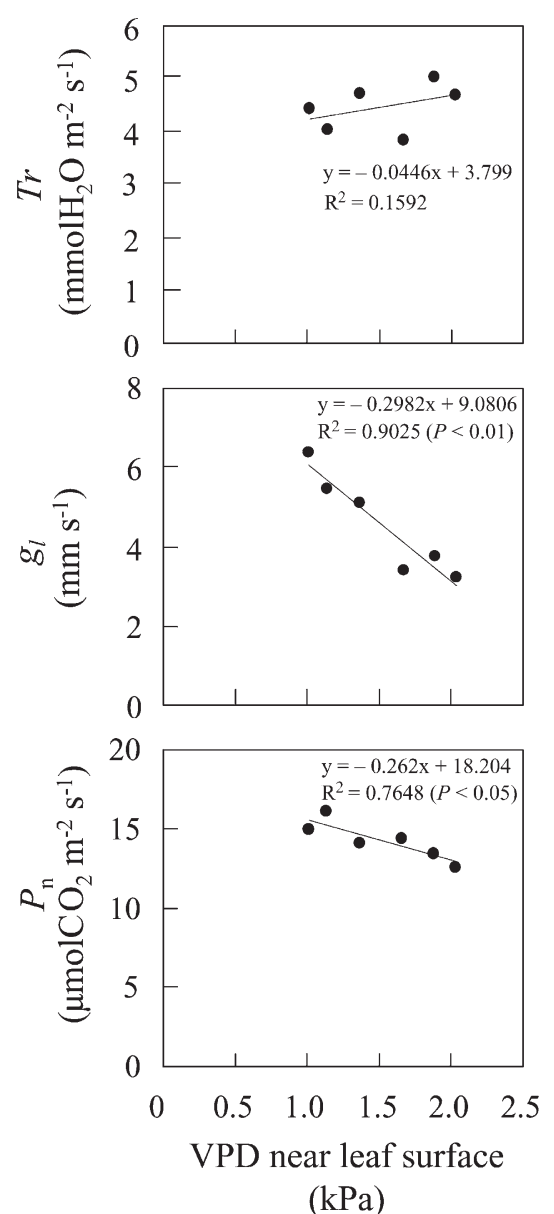

Fig. 6. Effects of vapor-pressure deficit (VPD) near the leaf surface on leaf conductance $\left(g_{1}\right)$ and net photosynthetic rate $\left(P_{\mathrm{n}}\right)$ of cucumber seedlings at high VPD (average 60 to $90 \mathrm{~min}$ after raising VPD).

relatively low compared with the plant community in commercial production. If measuring was conducted for much higher LAI, $P_{\mathrm{n}}$ per leaf area would be decreased by intershading and inhabitation of gas exchange by developing a boundary layer. Mitigating the inhibition of $P_{\mathrm{n}}$ under high plant density probably occurs in only cases in which stomatal closure resulting from high VDP affects a limiting factor of photosynthesis before other factors.

\section{Literature Cited}

Bunce, J.A. 1984. Effects of humidity on photosynthesis. J. Expt. Bot. 35:1245-1251.
Kim, Y.H., T. Kozai, C. Kubota, and Y. Kitaya. 1996a. Design of a wind tunnel for plug seedlings production under artificial lighting. Acta Hort. 440:153-158.

Kim, Y.H., T. Kozai, C. Kubota, and Y. Kitaya. 1996b. Effects of air current speeds on the microclimate of plug stand under artificial lighting. Acta Hort. 440:354-359.

Kitaya, Y., T. Shibuya, T. Kozai, and C. Kubota. 1998. Effects of light intensity and air velocity on air temperature, water vapor pressure and $\mathrm{CO}_{2}$ concentration inside a plant canopy under an artificial lighting condition. Life Support Biosph. Sci. 5:199-203.

Kitaya, Y., T. Shibuya, M. Yoshida, and M. Kiyota. 2004. Effects of air current speed on photosynthesis of plant canopies under elevated $\mathrm{CO}_{2}$ levels in a plant culture system. 2004. Adv. Space Res. 34:1466-1469.

Kitaya, Y., J. Tsuruyama, M. Kawai, T. Shibuya, and M. Kiyota. 2000. Effects of air current on transpiration and net photosynthetic rates of plants in closed plant production, p. 83-90. In: Kubota, C. and C. Chun (eds.). Transplant production in the 21 st century. Kluwer Academic Publishers, Dordrecht, The Netherlands.

Kitaya, Y., J. Tsuruyama, T. Shibuya, M. Yoshida, and M. Kiyota. 2002. Effects of air current speed on gas exchange in plant leaves and plant canopies. Adv. Space Res. 31:177-182.

Monteith, J. and M. Unsworth. Principles of environmental physics. 3rd Ed. 2008. Elsevier, Burlington, MA.

Shibuya, T. and T. Kozai. 1998. Effects of air current speed on net photosynthetic and evapotranspiration rates of a tomato plug sheet under artificial light. Environ. Control in Biol. 36:131136 [Japanese text with English abstract].

Shibuya, T. and T. Kozai. 2001. Light-use and water-use efficiencies of tomato plug sheets in the greenhouse. Environ. Control in Biol. 39:35-41 [Japanese text with English abstract].

Shibuya, T., R. Terakura, Y. Kitaya, and M. Kiyota. 2006. Effects of low relative humidity and illumination on leaf water status of cucumber seedlings and growth of harvested cuttings. HortScience 41:410-413.

Shibuya, T., R. Terakura, and M. Kiyota. 2003. Effects of short-term treatment of air humidity on growth and transpiration characteristics of cucumber seedlings and on growth of their cuttings. Environ. Control in Biol. 41:347352 [Japanese text with English abstract].

Woledge, J., J.A. Bunce, and V. Tewson. 1989. The effect of air humidity on photosynthesis of ryegrass and white clover at three temperatures. Ann. Bot. (Lond.) 63:271-279.

Yabuki, K. 2004. Photosynthetic rate and dynamic environment. Kluwer Academic Publishers, Dordrecht, The Netherlands.

Yabuki, K., J. Tamura, Y. Oda, and T. Imazu. 1967. The effect of carbon dioxide concentration of atmospheric on growth of leaf beet with various plant densities. J. Agr. Met. 23:25-29 [Japanese text with English abstract]. 